\title{
A Case Report on Rhinocerebral Mucormycosis of a Post COVID-19 Diabetic Patient
}

\section{Col. Md. Monirul Hoque ${ }^{1}$, Lt. Col. Khandaker Rokshana Akhter², Maj. Mehedi Hassan Shourov $^{1}$, Maj. SM Hasib Kausar ${ }^{1}$}

${ }^{1}$ Department of Pathology, AFIP, Dhaka Cantonment, Bangladesh.

${ }^{2}$ Department of Biochemistry, Armed Forces Medical College, Dhaka Cantonment, Bangladesh.

\begin{abstract}
Mucormycosis is an invasive fungal infection caused by different saprophytic environmental fungus occurring predominantly among immunosuppressed patients. Coronavirus disease 2019 (COVID-19) itself and its treatment with immunosuppressive drugs and oxygen delivery systems are setting the scenes for opportunistic and co-infections with fungus. An elderly Bangladeshi woman with background of diabetes mellitus, hypertension and COVID-19, presented with the complaints of nasal obstruction, yellowish nasal discharge, hairing impairment and loosening of teeth. An early diagnosis combined with medical and surgical intervention can decrease mortality and morbidity. This is one of the few cases of post-COVID mucormycosis reported from Bangladesh.
\end{abstract}

Key word: Rhinocerebral Mucormycosis, COVID-19

\section{Introduction}

Mucormycosis (zygomycosis) is a rare opportunistic fungal infection caused by fungi belonging to the order Mucorales and the family Mucoraceae ${ }^{1}$. It is the third most common angioinvasive fungal infection after candidiasis and aspergillosis ${ }^{2}$. It usually affects the immunocompromised individuals and rarely causes disease in apparently healthy individuals ${ }^{3}$.

In the immunocompromised host, mucormycosis infection results from rapid proliferation and invasion of fungal organisms in deeper tissues ${ }^{4}$. The major risk factors for mucormycosis include uncontrolled diabetes mellitus with ketoacidosis, other metabolic acidosis, corticosteroid therapy, organ or bone marrow transplantation, neutropenia, trauma and burns, hematologic malignancy (leukaemia, lymphoma, etc) and iron chelation therapy in patients receiving hemodialysis ${ }^{5-7}$.

Coronavirus disease 2019 (COVID-19) itself, its treatment with corticosteroids and their immunomodulators, invasive and non-invasive ventilator supports and other oxygen delivery systems, prolonged hospital stay and comorbidities,

Correspondence:

Col. Md. Monirul Hoque

SUP, MBBS, MCPS, DCP, FCPS (Microbiology)

Classified Specialist in Pathology

AFIP, Dhaka Cantonment

Mobile: 0171633601, Email: address:mmhoque21@yahoo.com all are setting the scenarios for opportunistic infections and co-infections with fungus. In case of diabetic ketoacidosis, in high blood glucose and acidic $\mathrm{PH}$, phagocytes become dysfunctional and their chemotaxis and intracellular killing mechanisms become impaired ${ }^{8}$. Infection starts with inhalation of spores through the nose or mouth or even through a skin laceration followed by spread to the paranasal sinuses and subsequently to the orbit, meninges and brain by direct extension ${ }^{9}$. Early identification of the disease and aggressive and prompt medical and surgical interventions are required to prevent the high morbidity and mortality associated with this disease process ${ }^{10}$.

\section{Case report}

A 56-year-old Muslim housewife, a known case of uncontrolled diabetes mellitus, hypertension and previous SARS-CoV-2 infection with pneumonia reported to ENT department of Combined Military Hospital, Dhaka Cantonment on 27 May, 2021 with the complaints of nasal obstruction and yellowish nasal discharge for last 01 month. These complaints developed after 15 days of recovery from Covid-19 infection. She also complained of gradual loosening of teeth and impairment of hearing for same duration. On examination, she was ill looking and moderately anaemic. Nasal endoscopy revealed left sided DNS along with blackish crusts found in the mucous membrane of the nasal cavity, nasopharynx, posterior 
nasal septum and also in the soft palate. There was an oronasal fistula with loosening of teeth at upper jaw. Hearing tests revealed conductive type of hearing loss in both sides. Other systemic examinations were normal.

Her related investigations were sent to Armed Forces Institute of Pathology, Dhaka Cantonment. Her Complete blood count showed: Haemoglobin $7.60 \mathrm{gm} / \mathrm{dl}$, total White Blood Cells $15.8 \times 10 \% / \mathrm{L}$ with Neutrophil $72 \%$, platelet count $578 \times 10^{9} / \mathrm{L}$, ESR $60 \mathrm{~mm}$ in $1^{\text {st }} \mathrm{hr}$.

Urine R/M/E showed : Albumin ++, Glucose++, ketone body: absent.

Other biochemical markers were FBS $12.7 \mathrm{mmol} / \mathrm{l}$, HbA1c 8.1\%. Her Serum creatinine was $2.2 \mathrm{mg} / \mathrm{dl}$. Her Serum TSH was $1.14 \mu \mathrm{IU} / \mathrm{ml}$, Serum iron profile: S. iron $31 \mu \mathrm{gm} / \mathrm{dl}$, S. Ferritin $342.5 \mathrm{ngm} / \mathrm{ml}$, TIBC $285 \mu \mathrm{gm} / \mathrm{dl}$. Her Serum electrolytes were $\mathrm{Na}^{+} 126 \mathrm{mmol} / \mathrm{l}, \mathrm{K}^{+} 2.3$ $\mathrm{mmol} / \mathrm{l}, \mathrm{Cl}^{-1} 100 \mathrm{mmol} / \mathrm{l}, \mathrm{HCO}_{3}{ }^{-} 22.1 \mathrm{mmol} / \mathrm{l}$.

Aseptically collected nasopharyngeal swab was examined under microscope. Wet film preparation, $\mathrm{KOH}$ preparation and Lactophenol cotton blue preparation showed sporangium with sporangiospores, broad (10-20 $\mu \mathrm{m})$, nonseptated, ribbon like hyphae with wide angle branching at irregular intervals morphologically resemble Mucor species. Culture in SDA media yielded the growth of fluffy, whitish to yellow colony of Mucor species after three days of incubation at $22^{\circ} \mathrm{C}$ in aerobic condition with optimum $\mathrm{PH}$. (Fig:1,2,3,4)

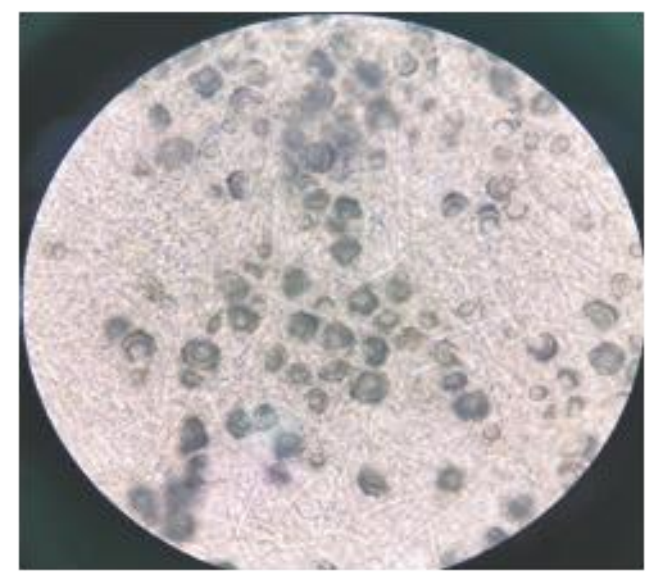

Fig 1: Broad,nonseptated,hyphae with sporangium $(\mathrm{KOH}$ preparation $40 \mathrm{X}$ )

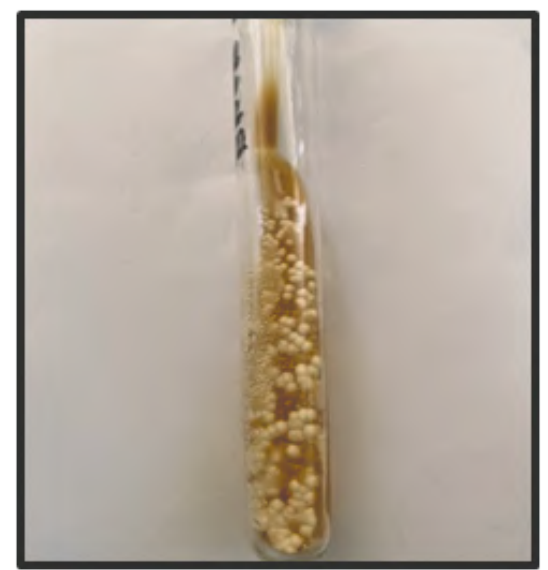

Fig 2: Whitish to yellow colony of Mucor species in SDA media

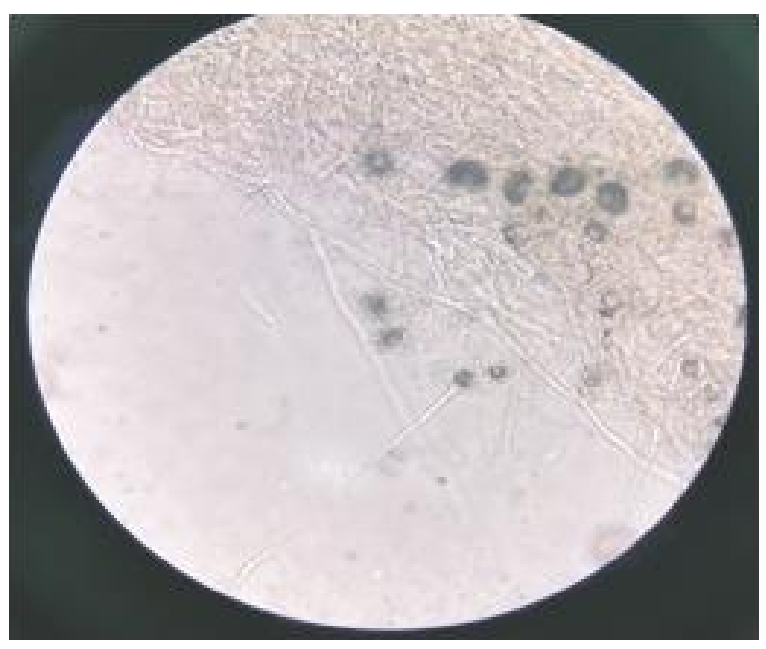

Fig 3: Wet film preparation from culture shows broad, nonseptated, ribbon like hyphae with wide angle branching at irregular intervals

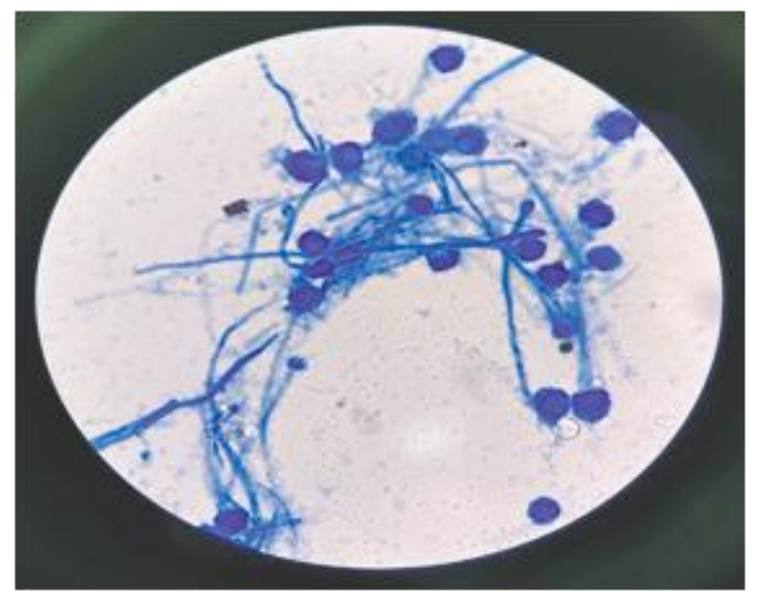

Fig 4: Staining by Lactophenol Cotton Blue shows wide angle branching hyphae with spore 
Histopathology of tissue from posterior nasal cavity revealed necroinflammatory exudates with fungal ball composed of hyphae and spores arranged in compact clusters which were positive for PAS stain (Fig 5).

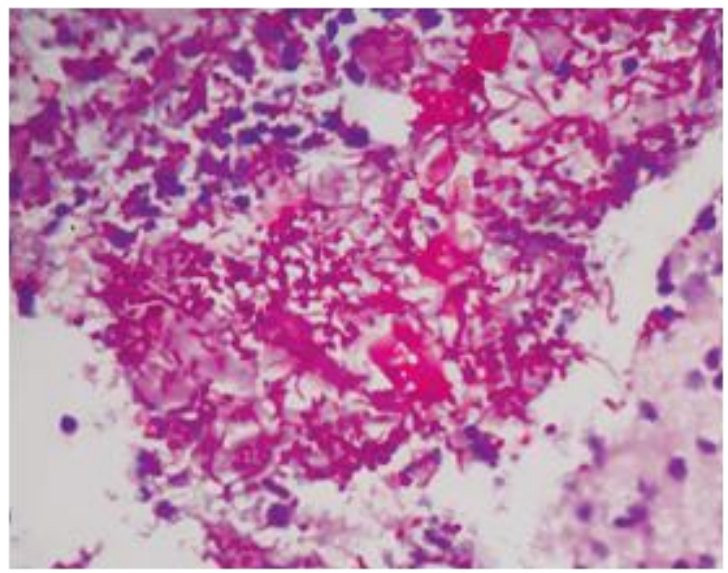

Fig 5: Positive PAS stain shows fungal ball composed of hyphae and spores

Spiral CT Scan of P.N.S were suggestive of fungal acute pan sinusitis with bony erosions at right and left alveolar process of maxilla and bilateral chronic mastoiditis (Fig 6). CT Scan of brain shows no significant changes.

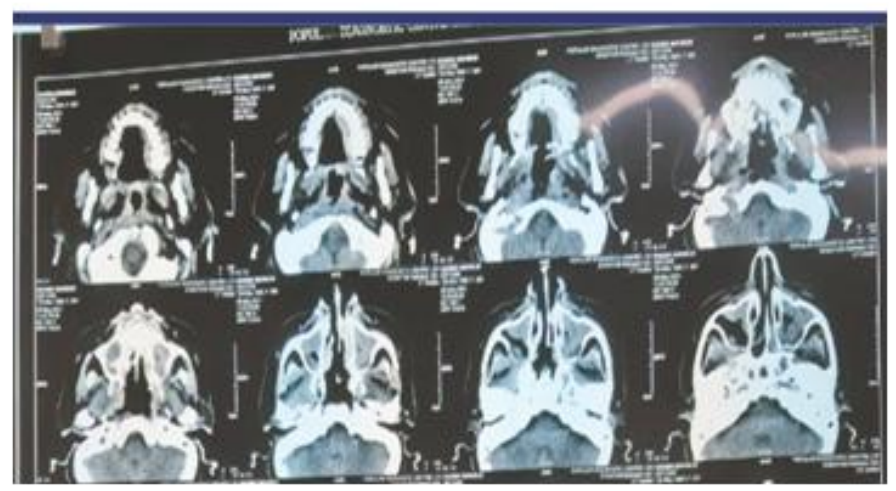

Fig 6: Spiral CT Scan of PNS shows pansinusitis with bony errosion

She was planned for surgical debridement under general anaesthesia followed by IV antifungal Liposomal Amphotericin B with other supportive therapy.

\section{Discussion}

Though mucormycosis is an uncommon disease, its prevalence in India is 70 times the world wide estimated rate ${ }^{11}$. So far 187 cases of COVID-19 associated mucormycosis have been reported in India during this pandemic ${ }^{12}$. No specific data is available in our country.
Studies have shown that diabetes mellitus alters the immunologic capability to resist mucormycosis through reduction of the phagocytic ability of granulocytes ${ }^{13}$. In addition, Rhizopus species thrive best in an acidic and glucose-rich environment. Differential diagnosis includes aspergillosis, histoplasmosis, paracoccidioidomycosis. A definitive diagnosis of mucormycosis can only be made by a biopsy and culturing the fungus in the laboratory ${ }^{14}$. Histologically, mucormycosis is characterized by extensive tissue necrosis, large fungal hyphae, which are nonseptate and have a ribbon-like appearance, with bodding and dichotomous branching ${ }^{15}$. However, in our case, the diagnosis was done immediately by wet film microscopy with aseptically collected specimen and further confirmed by culture in SDA media.

If mucormycosis is suspected, initial empirical therapy with antifungal drugs Amphotericin B should begin. Surgical debridement of the infected and necrotic tissue is the standard treatment along with the medical treatment. In our case we have started antifungal therapy with Amphotericin-B without delay which retard the further angioinvasion. Combined treatment increases the survival to $78 \%$ as compared to $57.5 \%$ with medical treatment alone. In the presence of intracranial extension, surgical debridement may not be possible and almost all the cases are fatal $^{16}$. Several surgical procedures have been described ranging from debridement of the mucosa, Caldwell-Luc surgery, medial maxillectomy, ethmoidectomy, sphenoidectomy, radical maxillectomy with orbital exenteration. The standard medical therapy is Amphotericin B in a dose of $1-1.5 \mathrm{mg} / \mathrm{kg} /$ day for several weeks depending upon the clinical response and degree of nephrotoxicity ${ }^{17}$. Other modalities of treatment tried are hyperbaric oxygen therapy and nasally nebulized Amphotericin B, oral Posaconazole ${ }^{18}$.

\section{Conclusion}

Early diagnosis by experienced microscopist, surgical debridement and antifungal therapy with Amphotericin B can reduce the mortality and morbidity of mucormycosis cases.

\section{Conflict of Interest}

The authors stated that there is no conflict of interest in this study. 


\section{Reference}

1. Viterbo S, Fasolis M, Garzino-Demo P, Griffa A, Boffano $\mathrm{P}$, Iaquinta $\mathrm{C}$, et al. Management and outcomes of three cases of rhinocerebral mucormycosis. Oral surgery, oral medicine, oral pathology, oral radiology, and endodontology. 2011 Dec 1;112(6): e69-74.

2. Torres-Narbona M, Guinea J, Muñoz P, Bouza E. Zygomycetes and zygomycosis in the new era of antifungal therapies. Revista espanola de quimioterapia: publicacion oficial de la Sociedad Espanola de Quimioterapia. 2007 Dec 1;20(4):375-86.

3. Goel S, Palaskar S, Shetty VP, Bhushan A. Rhinomaxillary mucormycosis with cerebral extension. Journal of oral and maxillofacial pathology. 2009 Jan;13(1):14..

4. Salisbury III PL, Caloss Jr R, Cruz JM, Powell BL, Cole R, Kohut RI. Mucormycosis of the mandible after dental extractions in a patient with acute myelogenous leukemia. Oral surgery, oral medicine, oral pathology, oral radiology, and endodontology. 1997 Mar 1;83(3):340-4.

5. Spellberg B, Edwards Jr J, Ibrahim A. Novel perspectives on mucormycosis: pathophysiology, presentation, and management. Clinical microbiology reviews. 2005 Jul;18(3):556-69.

6. Sugar AM. Agents of mucormycosis and related species. In: Mandell GL, Bennett JE, Dolin R, editors. Principles and practice of infectious diseases. 6th ed. Philadelphia, PA: Elsevier; 2005. p.2979

7. Ibrahim AS, Edwards JE, Filler SG. Zygomycosis. In: Dismukes WE, Pappas PG, Sobel JD, editors. Clinical mycology. New York, NY: Oxford University Press; 2003.pp.241-51.

8. Chinn RY, Diamond RD. Generation of chemotactic factors by Rhizopus oryzae in the presence and absence of serum: relationship to hyphal damage mediated by human neutrophils and effects of hyperglycemia and ketoacidosis. Infection and immunity. 1982 Dec;38(3):1123-9.
9. Mohindra S, Mohindra S, Gupta R, Bakshi J, Gupta SK. Rhinocerebral mucormycosis: the disease spectrum in 27 patients. Mycoses. 2007 Jul;50(4):290-6.

10. Bakathir AA. Mucormycosis of the jaw after dental extractions: two case reports. Sultan Qaboos University medical journal. 2006 Dec;6(2):77.

11. Chander J, Kaur M, Singla N, Punia RP, Singhal SK, Attri AK, et al. Mucormycosis: battle with the deadly enemy over a five-year period in India. Journal of fungi. 2018 Jun;4(2):46.

12. Patel A, Agarwal R, Rudramurthy SM, Shevkani M, Xess I, Sharma R, et al. Multicenter epidemiologic study of coronavirus disease-associated mucormycosis, India. Emerging infectious diseases. 2021 Sep;27(9):2349.

13. Lador N, Polacheck I, Gural A, Sanatski E, Garfunkel A. A trifungal infection of the mandible: Case report and literature review. Oral surgery, oral medicine, oral pathology, oral radiology, and endodontics. 2006;101:451-6.

14. Sugar AM. Mucormycosis. Clinical infectious diseases. 1992;14:126-9

15. Sapp JP, Eversole LR, Wysocki GP. Contemporary Oral and Maxillofacial Pathology. St Louis: Mosby; 1997.p.237-4

16. Munir N, Jones NS. Rhinocerebral mucormycosis with orbital and intracranial extension: a case report and review of optimum management. The Journal of laryngology \& otology. 2007 Feb;121(2):192-5.

17. Yadav SPS, Goel AK. Rhino-orbital mucormycosis-A case report. International journal of pediatric otolaryngology. 2010;5:9-12

18. Scheckenbach K, Cornely O, Hoffmann TK, Engers R, Bier H, Chaker A, et al. Emerging therapeutic options in fulminant invasive rhinocerebral mucormycosis. Auris nasus larynx. 2010 Jun 1;37(3):322-8. 